\title{
Evaluating a Pedagogical Approach to Promoting Academic Integrity in Higher Education: An Online Induction Program
}

Academic integrity is at the heart of excellent education. However, resources explaining the concept tend to be definition-driven, while using complex language and sometimes even an austere tone designed to discourage students from breaches. This study aims to evaluate a newly implemented online module at a UK University across two years, designed to improve students' understanding of concepts of academic integrity and practice. The module includes a range of interactive resources (e.g., gamified quizzes and e-booklets) and was made available to a large cohort of postgraduate students (400+). The study adopts a mixed-methods approach composed of three sequential phases involving first collecting students' views on existing academic integrity resources, then developing a range of new ones based on students' feedback to form the content of the module, and finally gathering students' evaluation on the newly created resources. Results illustrate a clear improvement in relation to the accessibility, usefulness and understandability of new resources. Results also highlight a remarkable increase in student confidence levels regarding academic integrity. Students also considered the new module as more appealing and informative. This paper offers a good example of a pedagogical approach aimed at promoting academic integrity in an innovative and engaging fashion.

Keywords: academic integrity, policy, practice, students, mixed-methods study

\section{Introduction}

Academic integrity is at the heart of excellent teaching and learning; it can be defined as a commitment and demonstration of honest and moral behaviour in an academic setting; it is applicable to both students and academic staff. Macfarlane, Zhang and Pun (2014) conduct a systematic literature review that shows research into academic integrity is often centred on unethical student behaviours, which may be either accidental or intentional (Walker and White 2014). For example, Greenberger et al. (2016) argue one of the most common breaches of academic integrity is plagiarism, caused by poor 
paraphrasing practices and incorrect referencing formats. At the other end of the spectrum is deliberate attempts at cheating, which range from buying, selling, or trading essays, to arranging for someone else to take an exam (Bretag et al. 2019; Awdry et al. 2021). However, research suggests the definition of academic integrity is not universally understood and is open to different interpretations; this may cause misunderstanding among staff and students, and ultimately lead to unethical academic behaviours (Gullifer and Tyson 2014).

Upholding academic integrity in higher education is vital for all stakeholders. Nevertheless, universities face increasing challenges related to breaches in student academic integrity, whether unintentional or deliberate (Mahmud and Ali, 2021). A key issue appears to be centred on aligning concepts of academic integrity, policies and processes, with teaching and learning practices. This study aims to design, implement and evaluate an online academic integrity module with resources tailored to student needs. This paper presents results of qualitative and quantitative evaluation data, aiming to inform current teaching practice related to academic integrity in higher education.

\section{The Challenges of Student Academic Integrity Development in Higher}

\section{Education}

Existing literature highlights many barriers to the teaching of academic integrity within the higher education sector. One significant barrier appears to be complex terminology and unclear processes associated with breaches in academic integrity (Bretag et al. 2019; Ransome et al 2018). Another barrier is often caused by competing views across cultures of what constitutes academic integrity and unethical academic conduct (Khanal and Gaulee 2019; Palmer et al. 2017; Zhang et al. 2014), or even across academic institutions within a single national culture (Bretag et al. 2014; Walker and White 2014). This may result in mixed messages to students who enter new institutions with 
misaligned prior knowledge of these concepts in relation to institutional requirements (Bertram Gallant and Kalichman 2011). Other barriers that have been frequently mentioned in the literature include language (e.g. unfamiliarity with academic writing style) (Newton 2016) and cultural barriers (Bista 2011), which are often experienced by students whose first language is not English (Fass-Holmes 2017; Jian et al 2019). For example, Mahmud et al. (2019) highlight significant differences in student perceptions of academic integrity in relation to the UK and Eastern European countries, advocating that academic integrity policies should be considerate of national cultures. Clough, Willett, and Lim (2015) interviewed 30 Unfair Means officers, finding that unfair means, or breaches of academic integrity in essay-based assignments, are more common among non-native English speakers. Other researcher highlights a danger in the oversimplistic view that international students cheat due to culturally diverse values (Bretag et al. 2019), potentially leading to staff being biased towards them (Zhao and Kung 2021).

\section{A Theoretical Framework of Academic Integrity}

Recent research developments heighten the requirement for a holistic approach in promoting academic integrity, including the establishment of clearly defined academic integrity, acknowledging the responsibility of all stakeholders. Löfström et al. (2015) observe that there are mismatches in the perspectives of teachers and students regarding responsibility for upholding academic integrity standards in universities. Bealle (2017) argues that students, as those most affected by academic integrity policies, tend to become passive recipients of change initiatives rather than the drivers; he emphasises how the responsibility of maintaining academic integrity at an educational institution should not fall only to students, but also should involve all stakeholders. In the same 
vein, Bretag et al. (2014) call for universities to move beyond mere information provision towards a more holistic approach, aiming to engage all parties throughout the process.

To achieve a holistic approach to academic integrity, a theoretical model is necessary to guide universities. Bretag et al. $(2014,2011)$ propose exemplary academic integrity policy provision as consisting of five core aspects: easy access to academic integrity related policies (access), a consistent communication strategy on academic integrity values and practices (approach), clearly outlined responsibilities of all stakeholders (responsibilities), a detailed description of breaches, processes, and consequences (detail), proactive and embedded support to engage students in the policies (support). According to Bretag et al. (2014), approach and responsibility aspects often fall within the institution's domain; the other three aspects (access, detail and support) are addressed at a departmental level; this model provides an effective basis for teaching and researching academic integrity at universities (Mahmud et al. 2019).

\section{Promoting Academic Integrity in Higher Education: Towards a Pedagogical Approach}

Existing research recognises two dominant approaches to preventing student Academic Integrity breaches: punitive and educative. Richard et al. $(2016,243)$ suggest a punitive approach aims to 'deter students from committing breaches through the threat of penalties', whereas an educative approach aims to 'reduce the likelihood of students committing breaches by providing them with relevant skills and knowledge'. Conversely, Walker and White (2014) propose two plagiarism prevention models, the 'ethical' model, emphasising students' active role in adhering to the academic integrity code of conduct, and the 'pedagogical model', focused on equipping students with 
appropriate academic skills. Although a punitive approach can communicate to students that plagiarism has serious consequences, research indicates that this approach alone is not sufficient to reduce cheating (Miller et al. 2011; Sun and Hu, 2020). Zhao and Kung (2021) highlight for universities the importance of adopting an educative (pedagogical) approach to provide consistent and continuous teaching for students related to academic integrity before applying a more severe punitive or judicial approach as more serious cases, such as contract cheating, are found.

Following a pedagogical approach, a number of researchers have highlighted the value of an early intervention strategy that is positive, proactive, engaging and continuous to help students' academic integrity development when entering a new department (Bista 2011; Bretag et al. 2014; Belter and du Pré 2009; Walker and White 2014; Newton 2016). For example, Bertram Gallant $(2008,89)$ suggests that universities should shift the focus from enforcing rules and policies to ensuring students learn about academic integrity, fostering 'a learning-oriented environment, improving instruction, and enhancing institutional support for teaching and learning'. Pàmies et al. (2020) argue that universities need to take more responsibility for educating their students about plagiarism and explaining how to properly cite sources.

Research suggests that there has been improvement in university commitment to addressing academic integrity issues (Burbidge and Hamer 2020); however, the implementation process within teaching remains unsatisfactory (Gottardello and Karabag 2020; Bretag et al. 2014). Christie et al. (2013) report a disparity between academic integrity promotion and actual teaching practice which assimilates academic integrity in the classroom. Particularly, postgraduate students are less informed of academic integrity policies (Fatemi and Saito 2020), and often left underprepared for research-based academic assignments (Mahmud and Bretag 2013). Therefore, it is of 
vital importance to help students develop a clear understanding of academic integrity (Tatum et al. 2017).

A number of educative strategies have proven effective. For example, researchers have demonstrated that educational initiatives, such as online modules that focus on academic integrity, can positively impact students' attitudes, reducing potentially unethical behaviours (Bealle 2017; Belter and du Pré 2009; Ballard 2013; Sefcik et al. 2020; Du 2020). Boehm, Justice and Weeks (2009) suggest that providing clear definitions with specific examples of what constitutes unethical behaviours can effectively prevent academic integrity breaches. Bretag et al. (2014) argue that education resources on academic integrity should be engaging and creative; for example, using storytelling and narrations. Furthermore, they suggest that regular email reminders should be incorporated, providing ongoing support for students. Macfarlane et al. (2014) propose the provision of educative resources, and design of student-centred activities, should be informed by student feedback and tailored towards student needs. However, little empirical research directly addresses the topic of academic integrity through the use of interventional strategies, such as academic integrity courses (Sotiriadou et al. 2020; Elander et al. 2010).

\section{Research Aim and Objectives}

This research aims to evaluate the effectiveness of a newly created module in enhancing students' understanding of academic integrity concepts, policies and practices. The module is designed to include a suite of interactive activities and engaging materials which are based on student feedback and suggestions.

The objectives of research are: 
(1) To explore students' needs for academic integrity related activities and resources.

(2) To design and implement induction activities and learning resources to support students' academic integrity development based on student feedback.

(3) To examine the effectiveness of induction activities in enhancing students' awareness and knowledge of academic integrity.

\section{Materials and Methods}

\section{Research Design}

This study adopted a mixed-methods inductive approach, conducted in a post-graduate school at a research-intensive university in the UK. Both quantitative and qualitative data were gathered across the course of a sequential study composed of three main phases (Figure 1).

[Please insert Figure 1 here] Figure 1. Phases of the study.

In phase 1 (January-February 2020), two focus group interviews and a questionnaire were conducted with students, exploring views and understanding of academic integrity. At the focus groups, in-depth discussions were carried out with a sample of seven students from different programmes (Undergraduate and Postgraduate) of study and genders and covering a range of cultures (Home, European and Overseas students). Students were asked questions around three main areas: their general understanding of the principles and concepts of academic integrity, the support received to date with respect to academic integrity, and their views on the existing academic integrity resources. Thematic Analysis was applied to focus group data to support a deeper understanding of participants' individual circumstances and experiences of 
academic integrity (Braun and Clarke 2006). This approach is commonly applied in qualitative research designs, attempting to identify differences and similarities in experiences. Analysis of focus group discussions informed the design of a questionnaire, covering aspects related to student understanding of academic integrity, approaches to searching for academic integrity related resources, their perception and needs in terms of content and style of the academic integrity resources available, and suggestions to make them more relevant and accessible. Furthermore, it listed eight aspects of existing academic integrity resources (reliable, comprehensive, easy to use, useful, credible, convenient and accessible, easy to understand and trustworthy); students were asked to rate on a Likert scale from 1 (completely disagree) to 5 (completely agree).

In phase 2 (April-September 2020), authors developed a range of resources based on student feedback from phase 1, enhancing accessibility, detailed contents, and support regarding academic integrity education within the department. These resources, collated under the term 'Academic Integrity Activities', were hosted in an online Blackboard module which was accessible to students. The package included an interactive video recording with embedded questions on academic integrity and other key information for students; a gamified academic integrity quiz with 20 scenario questions; a FAQs document related to common academic integrity related queries; an electronic booklet (e-booklet) containing detailed examples of commonly occurring academic integrity problems and their solutions (see Figure 2 for screenshots examples of some of the resources). Students were asked to access and familiarise themselves with the resources and undertake the quiz as part of induction activities at the beginning of the academic year. Additionally, a series of four online, synchronous drop-in sessions were offered to students, spread throughout the Autumn Semester; the sessions were 
student-led, covering topics suggested by students via online polls which informed the design and content of the next session.

[Please insert Figure 2 here] Figure 2. Pictured left is a representative example of a question included in the 20-question quiz; on the right is a screenshot of a page from the e-booklet.

In phase 3 (September-November 2020), a second questionnaire was distributed to obtain students' feedback regarding the newly created academic integrity resources. The questionnaire included both closed and open-ended questions. To support continuity and comparability of results, the questionnaire contained most questions initially asked in phase 1, including the rating of eight key aspects of academic integrity. However, additional open-ended questions were asked regarding the timeliness of support in the academic year, and in what ways the revised resources could be further improved and promoted to students. Furthermore, respondents were asked about their confidence in relation to academic integrity related concepts before and after use of the new resources. Phases 1 and 3 sought and received ethical approval from the University of Sheffield Research Ethics Committee.

\section{Participants}

Participants in phase 1 were selected according to the following: participants in the two focus groups were postgraduate students from the same academic department; respondents to the questionnaire were both undergraduate and postgraduate students from the wider student body at a British University. Research was advertised via email and on the University noticeboard; only volunteer students participated. No incentives were offered. Participants in phase 3 were postgraduate taught (PGT) students enrolled 
in varied programmes of study, but working within the same department; all accessed the induction module containing phase 2 induction activities. The feedback questionnaire was included as the last task for completion in the induction activities. The rationale behind the inclusion of only PGT students from an academic department was based on the fact all resources created in phase 2 were designed specifically for this cohort. Additionally, research suggests this cohort of students require more support compared with UG students, due to the short length of their study (Mahmud and Bretag 2013).

\section{Data Analysis}

Descriptive statistics, paired sample t-tests, comparison of means, and N-1 Chi-squared tests, were used to analyse quantitative data derived from the two questionnaires using IBM SPSS 26. Qualitative data from the focus groups, and open-ended questions from the two questionnaires, were manually transcribed and analysed using the six-step approach to thematic analysis established by Braun and Clarke (2006).

\section{Results}

In phase 1, 39 students from a variety of undergraduate and postgraduate programmes of study, participated in research; this included two focus groups $(\mathrm{n}=7)$, and a smallscale survey $(n=32)$, to inform the design of academic integrity related activities. Initial analysis of student feedback was conducted and used for planning of phase 2 .

Focus group interview results from phase 1 highlight three areas for improvements: academic integrity-related terminology, delivery formats, and tone of communication. Students found the terminology around academic integrity tended to be complex and less engaging; they recommended academic integrity information should 
include "real examples" with analysis, and "scenario questions" that are easy to follow and appear relatable:

"The definitions are ok, but I would have liked more examples, practical scenarios so that it's easier to know what to avoid" (Focus group participant 1, China, Female)

They also recommended the content of academic integrity should be delivered in a more engaging manner, suggesting interactive videos, humorous print/online brochures, and drop-in sessions for Q\&A:

"Maybe print brochures and include them in the welcome pack, or draw some comics/make short videos (better if humorous)" (Focus group participant 5, China, Female)

Additionally, students found that the tone of academic integrity related communications tended to be 'scary'. They expressed a preference towards a more neutral or supportive tone:

"I hope there could be a better way to approach this issue, such as the officer should hold a neutral ground and try to guide and explain to students of their mistakes and not try to make them feel ashamed of what happened" (Focus group participant 3, China, Male)

\section{Pre and post-intervention quantitative data analysis}

In the sample from phase 1 , ranging in age from 18 to $58,2 / 3$ of the students were female (66.7\%) and almost half (46.2\%) undergraduate, domestic students (Table 1). The seven students participating in the two focus groups were four females and three males; all held international student status. Again $2 / 3$ of the students in this sample were aware of academic integrity concepts prior to their arrival at university. However, the remaining ones did not, or were unsure. In phase 3 (Table 1), 361 PGT students from 
one academic department completed academic integrity activities, representing a completion rate of $81 \%$; 130 returned the feedback questionnaire (response rate 29\%). Similar to phase 1 , the majority of respondents were female $(60.8 \%)$. However, this time a higher proportion of overseas students (69.2\%), in comparison to phase 1 , completed the survey; this is in line with the standard composition of the academic department student body. In terms of initial awareness of concepts of academic integrity, $76.2 \%$ reported a clear understanding prior to joining the department. Of those reporting no awareness of academic integrity, $74 \%$ were non-domestic students.

[Please insert table 1 here] Table 1. Demographic characteristics of the students participating in the research.

As described in Materials and Methods, for comparative purposes, students participating in phases 1 and 3 were asked to rate the same eight aspects of academic integrity resources on a Likert scale from 1 (completely disagree) to 5 (completely agree). Such aspects and their ratings before (phase 1) and after (phase 3) the design of the suite of new academic integrity resources are summarised in Figure 3. A steady improvement in all aspects has been observed from the January 2020 (phase 1) to November 2020 questionnaire (phase 3), demonstrating an overall positive reception to the new academic integrity material that was developed in phase 2.

[Please insert Figure 3 here] Figure 3. Mean values of key aspects of academic integrity resources before (phase 1) and after (phase 3 ) implementation of induction tasks.

Paired sample t-tests conducted on data demonstrate the way new induction tasks have the largest statistically significant impact on how the perceived usefulness of the resources (from a mean of 3.79 in phase 1 , to 4.27 in phase $3, p=0.028 ; t=2.210$ ) and their understandability (from a mean of 3.62 in phase 1 to 4.09 in phase $3, p=0.031$; $t=2.168$ ). Aspects of trustworthiness and credibility increased least, from a mean of 4.10 
to 4.20 for trustworthiness and 4.10 to 4.14 for credibility in phase 1 and phase 3 respectively. However, these had previously been the highest ranked aspects to begin with.

Exploring the same academic integrity aspects in terms of proportion of students rating them 4 (agree) and 5 (completely agree), illustrates results that are similar. However, the differences between phase 1 and 3 perceptions are more striking; $17.1 \%$ more students agreed that the new resources were useful, and $28.5 \%$ more agreed that they were easy to understand.

Students were then asked whether they considered the online Academic Integrity module as adequate to support their learning. The vast majority of students $(85 \%)$ found the module adequate; the remaining $12 \%$ of students answered 'maybe' and a very small percentage of students (3\%) considered the module as inadequate (Figure 4). It is notable that this feedback was received from postgraduate students who attend 1-year programmes, and therefore do not have access to previous years' resources or modules. Furthermore, the study was conducted prior to students' first assessed submissions; it was not possible to gauge their actual understanding of academic integrity principles.

[Please insert Figure 4 here] Figure 4. Students' opinions of whether the new Academic Integrity resources were adequate for learning.

In the phase 3 questionnaire, students were asked to rate their confidence regarding academic integrity concepts prior to and following the use of the new resources on a Likert scale from 1 (very worried) to 5 (very confident). Results show that only $26 \%$ of respondents (overall mean=3.04) were confident (rated 4 and 5) before using resources. However, this percentage increased to $60.6 \%$ (overall mean $=3.70$, $\mathrm{p}<0.001)$ after use. Nevertheless, despite the considerable increase in positive 
perceptions recorded in phase 3 , around $70 \%$ of respondents still believed resources have the potential to be improved further (Figure 5).

[Please insert Figure 5 here] Figure 5. Students' opinions of whether the new Academic Integrity resources could be further improved.

In the phase 3 questionnaire, students were asked to indicate which of the new academic integrity resources they found most useful; they could select more than one option when answering this question (Figure 6).

[Please insert Figure 6 here] Figure 6. New academic integrity resources as ranked by perceived usefulness (according to student feedback).

All resources were well received; however, the e-booklet scored particularly highly $(41.1 \%)$, perhaps due to the attractiveness of its innovative format, one not normally used to deliver academic integrity content; this was followed by the scenariobased 20-question quiz (36.7\%) and the FAQ document (35.2\%). A small percentage of students $(6.3 \%)$ reported none of the resources as useful. However, this could be due to poor familiarity with the platform (Blackboard) in which they are embedded. Live dropin sessions were not included in the list, as the questionnaire was distributed before the sessions took place. Nevertheless, their utility was demonstrated in the consistently high number of students (over 170 in each one) attending them.

\section{Analysis of open-text data in phase 3}

Open-text questions were included in the phase 3 questionnaire, allowing students to elaborate on their view of phase 2 resources and provide suggestions for future improvement. Qualitative data suggests students highly valued the resources 
provided to them, which helped them develop an enhanced understanding of academic integrity concepts and varied types of breaches in academic integrity:

"I didn't expect to get such a detailed (and not boring) introduction on this subject." (Participant 28, Switzerland \& France, Male)

"I have attended lectures of [anonymised], their experience with students who used unfair means (knowingly or unknowingly) helped me a lot. Before that, I know just the theory behind unfair means but after examples, I realized that I do not know much about unfair means in practice." (Participant 114, India, Male) "I didn't know that there were so many sorts of unfair means, I thought plagiarism was the only sort so that is useful to know." (Participant 106, UK, Female)

Two main themes were identified in qualitative data, corresponding to the Academic Integrity Model (Bretag et al. 2014) in the aspects of access and support of academic integrity resources.

\section{Structured and accessible academic integrity materials}

Regarding access to academic integrity related resources and support, students reported that having all resources in one virtual space as very helpful. For example, having a single online module regarding academic integrity throughout their study allowed them to learn about academic integrity at the beginning of their studies, revisiting resources when they need them at a later stage, such as before coursework submission periods:

"Everything I need to establish what falls or doesn't under Academic Integrity can be found in the folders; which has been helpful." (Participant 31, Netherlands, Female)

"I appreciated the introductory material, and know where to go for more information/guidance when I need it." (Participant 37, UK, Male)

However, students also expressed a preference towards clear structure of the resources, avoiding information repetition or cognitive overload. Most suggestions for 
improvement related to making resources organised in a clear fashion on Blackboard. Many students who had not used Blackboard prior to joining the university found it challenging to navigate and struggled to follow material, particularly at the beginning of the academic year. Student recommendations include walkthrough videos, explaining the overall structure of resources, brief videos with clearly labelled topics, and a navigation side panel in the online space:

\footnotetext{
"The Blackboard software can be overwhelming as a new user. Clearer signposting to resources in general may improve access to learning materials." (Participant 8 , UK, Male)

"They are fine, though I think it needs to be streamlined a bit. There is a lot of content, and some of it is a bit repetitive. There should just be an explanatory video and then the quiz." (Participant 23, UK, Male)

"Include in the side panel on the main course page on Blackboard along with the Induction tasks." (Participate 31, UK, Female)

"I guess creating the whole information in form of some movie, while sticking only to the topic, would be more beneficial. And keeping the length of the videos small or making different videos for each topic...would make it easy to search in hour of need. E.g. a student might suddenly get confused about something and search for an answer, but for that he'll have to go through the whole video." (Participant 29, India, Female)
}

These recommendations will be taken into consideration in future modifications of resources.

\section{Engaging contents and mixed resources formats}

When considering the format of academic integrity related resources and support, students welcomed the variety in means of delivery (videos, e-booklets, gamified quizzes with cartoons, FAQs, and online drop-ins) finding them particularly engaging. Students made very positive comments regarding the online quizzes, which contain cartoon scenarios stories based on real cases. According to students, real examples 
support comprehension of academic integrity concepts better, connecting them with practices. The creative use of cartoons enhanced student engagement, inspiring students to use similar techniques in their studies:

\footnotetext{
"Gave overview of what unfair means (academic integrity) are...the quiz to test our understanding - made it more fun, and meant it wasn't just documents that we had to read." (Participant 27, UK, Female)

"The information being conveyed in different formats has made it clear to understand and retain.” (Participant 44, UK, Female)

"I particularly liked the quiz as it made me reread things in depth to get the right answer and it was real life examples rather than just pages of theory." (Participant 44, UK, Female)

"I liked the idea of presenting doodles in each question. I never felt bored because of them and I think I can use this idea of creating doodles." (Participant 25, India, Male)
}

Physical copies and printable versions were also frequently mentioned by students in comments. Although students prefer the mixed format provided by online resources, they suggested that physical copies, or downloadable materials, should be made available for students as an additional option:

"Email some of the documentation out because for the first couple of weeks I was still trying to get the hang of Blackboard and its multiple folders/files." (Participant 31, Netherlands, Female)

"The booklet of plagiarism is only available online, it's better if we can download it." (Participant 28, China, Female)

This goal could be easily achieved by sending regular email reminders to students with links to online resources and PDF attachments.

\section{Discussion}

This paper adopted a pedagogical approach to promoting academic integrity to students 
in higher education (Richard et al. 2017; Walker and White 2014). The study aims to evaluate the effectiveness of a newly created academic integrity module in relation to enhancing student understanding of academic integrity concepts, policies, and practices. Tailored to students' needs (Macfarlane et al. 2014), a sequential three-phase study was undertaken, collecting initial student feedback (phase 1), implementing an online academic integrity module (phase 2), and conducting a post-hoc evaluation survey (phase 3). The project proved successful in supporting the development of students' academic integrity.

Consistent with existing literature, this research found that, compared with home students, international students are less likely to be informed about academic integrity policies before their arrival to academic departments (Bista 2011; Fatemi and Saito 2020). However, both home and international students participating in the study expressed a desire for academic departments to improve academic integrity resources, rather than using punishment and threats to prevent breaches. This fact highlights the importance of an educative intervention to support the academic integrity of students, with programmes for diverse student cohorts. The findings of this research revealed a main barrier to student understanding of academic integrity, definition-driven terminologies that lack concrete examples. This finding is consistent with the literature, suggesting that universities should avoid complex language related to academic integrity (Bretag et al. 2019) and go beyond providing statements and definitions of academic misconduct (Risquez et al. 2013).

Another barrier reported by participants is a lack of creative resources and an absence of supportive tone from academic departments; this discourages student engagement and understanding of academic integrity; this broadly corresponds to findings of Betram Gallant (2008), who stressed the importance of creating a supportive 
learning-oriented environment which fosters development in students' academic integrity.

According to findings of this research, an online academic integrity module has proven to be an effective intervention strategy for increasing student awareness and understanding of academic integrity related concepts, procedures, and policies. This is consistent with the literature suggesting academic integrity education programmes can positively influence student attitudes and reduce breaches of academic integrity (Sefcik et al. 2020; Greer et al. 2012; Obeid et al. 2017; Levine et al. 2018). Student participants in the research revealed preference towards academic integrity resources and support that is structured and easy to access at different stages of their study. Comparative data analysis revealed the online Academic Integrity module significantly enhanced various aspects of academic integrity resources, particularly aspects of usefulness and comprehension of student perspectives. Results also revealed a remarkable increase in levels of student confidence regarding their knowledge of concepts of academic integrity. This evidences the importance of establishing early intervention, and a continuously accessible online course which promotes academic integrity in higher education.

Findings highlight the importance of the use of mixed media when delivering academic integrity resources to enhance student engagement and understanding. Students showed a preference towards online academic integrity booklets with examples of detailed analysis of good and poor practice; this allowed them to learn about different types of breaches, and accords with the findings of Boehm et al. (2019), who showed clear examples of what constitutes unethical academic behaviour are able to help prevent academic integrity breaches. Furthermore, students appreciated cartooned scenarios, entailing storytelling, and praised the entertaining and relatable 
nature of these materials. Results here reflect those of Macfarlane et al. (2014), who highlighted the need to use engaging techniques, such as storytelling and narration, to teach academic integrity related topics.

\section{Limitations and future directions}

As most participants were postgraduate students with essay-based assignments of social science subjects, generalisability of the results is subject to certain limitations. A second limitation regards the possibility of response bias in research and the associated implications. Participating students knew research was evaluating a new module developed by the authors, and they may have wished to please them and give them what they thought they expected (i.e. positive perceptions of the new materials). The authors attempted to minimise this issue by asking a volunteer $\mathrm{PhD}$ student to moderate focus groups. However, it would have been impossible to minimise this possibility. A further limitation regards the small sample size of the phase 1 study, which may also contribute to reducing the generalizable nature of results. Future research may seek to focus on different subject disciplines, comparing results with the current study. Additionally, further studies may also explore the impact of such a module on students' long-term engagement with academic integrity practices.

\section{Conclusion}

The results of this study have important implications for higher education in respect of the design of academic integrity resources to support students' academic transition. They showcase the effectiveness of an online academic integrity module in engaging students with academic integrity concepts, policies, and procedures. Findings suggest the topic of academic integrity, which tends to be serious, has potential be delivered via entertaining and informative methods. In summary, findings suggest higher education 
sectors should adopt a pedagogical approach in promoting academic integrity through engaging and relatable educational resources.

\section{Disclosure statement}

No potential conflict of interest was reported by the authors.

\section{References}

Awdry, Rebecca, Phillip Dawson, and Wendy Sutherland-Smith. 2021. "Contract cheating: To legislate or not to legislate-is that the question?." Assessment \& Evaluation in Higher Education: 1-15. doi: 10.1080/02602938.2021.1957773.

Ballard, Iva B. 2013. “The Impact of an Academic Integrity Module and Turnitin ${ }^{\circledR}$ on Similarity Index Scores of Undergraduate Student Papers." Research in the Schools 20 (2) : 1-13. doi: 10.1080/00405841.2017.1283573.

Bealle, Penny.2017. "Community college academic integrity lessons that put research into practice." Theory Into Practice 56 (2): 144-155.

Belter, R.W., and A. du Pré. 2009. “A strategy to reduce plagiarism in an undergraduate course." Teaching of Psychology 36 (4): 257-261. doi:10.1080/00986280903173165.

Bertram Gallant, T. 2017. “Academic integrity as a teaching \& learning issue: From theory to practice." Theory Into Practice 56 (2): 88-94.doi: 10.1080/00405841.2017.1308173.

Bertram Gallant, T. 2008. Academic integrity in the twenty-first century: A teaching and learning imperative. San Francisco, CA: Jossey-Bass.

Bretag, T., R. Harper, M. Burton, C. Ellis, P. Newton, P. Rozenberg, S. Saddiqui, and K. van Haeringen. 2019. "Contract cheating: a survey of Australian university students." Studies in Higher Education 44 (11): 1837-1856. doi: 10.1080/03075079.2018.1462788.

Bretag, T., S. Mahmud, M. Wallace, R. Walker, C. James, M. Green, J. East, U. McGowan, and L. Partridge. 2011. "Core elements of exemplary academic integrity policy in Australian higher education.” International Journal for Educational Integrity, 7 (2): 3-12. doi: 10.21913/IJEI.v7i2.759. 
Bretag, T., S. Mahmud, M. Wallace, R. Walker, U. McGowan, J. East, M. Green, L. Partridge, and C. James. 2014. "“Teach us how to do it properly!' An Australian academic integrity student survey. "Studies in Higher Education 39 (7): 11501169. doi: 10.1080/03075079.2013.777406.

Bista, K.K. 2011. “Academic dishonesty among international students in higher education." To improve the academy 30 (1): 159-172. doi:10.1002/j.23344822.2011.tb00655.x.

Boehm, P.J., M. Justice, and S. Weeks. 2009. "Promoting academic integrity in higher education." The Community College Enterprise 15 (1): 45-61.

Burbidge, T., and R. Hamer. 2020. “Academic Honesty in the International Baccalaureate Diploma Programme: Student, Teacher, and School Perspectives." Journal of International Students 10 (2): 265-285. doi:10.32674/jis.v10i2.667.

Christie, B.A., KK. Miller, R. Cooke, and J.G. White. 2013. "Environmental Sustainability in Higher Education: How do Academics Teach?" Environmental Education Research 19 (3): 385-414. doi: 10.1080/13504622.2012.698598.

Clough, P., P. Willett, and J. Lim. 2015. "Unfair means: use cases beyond plagiarism." International Conference of the Cross-Language Evaluation Forum for European Languages 9283: 229-234. doi:10.1007/978-3-319-24027-5_21.

Du, Yao. 2020. "Evaluation of intervention on Chinese graduate students' understanding of textual plagiarism and skills at source referencing." Assessment \& Evaluation in Higher Education 45 (1): 14-29. doi:

10.1080/02602938.2019.1601680.

Elander, James, Gail Pittam, Joanne Lusher, Pauline Fox, and Nicola Payne. 2010. "Evaluation of an intervention to help students avoid unintentional plagiarism by improving their authorial identity." Assessment \& Evaluation in Higher Education 35 (2): 157-171. doi: 10.1080/02602930802687745.

Fass-Holmes, B. 2017. "International Students Reported for Academic Integrity Violations: Demographics, Retention, and Graduation." Journal of International Students 7,(3): 664-669. doi: 10.5281/zenodo.570026.

Fatemi, Ghazalossadat, and Eisuke Saito. 2020. "Unintentional plagiarism and academic integrity: The challenges and needs of postgraduate international students in 
Australia." Journal of Further and Higher Education 44 (10): 1305-1319. doi:10.1080/0309877X.2019.1683521.

Greenberger, S., R. Holbeck, J. Steele, and T. Dyer. 2016. "Plagiarism Due to Misunderstanding: Online Instructor Perceptions." Journal of the Scholarship of Teaching and Learning 16 (6): 72-84. doi: 10.14434/josotl.v16i6.20062.

Greer, Katie, Stephanie Swanberg, Mariela Hristova, Anne T. Switzer, Dominique Daniel, and Sherry Wynn Perdue. 2012. "Beyond the web tutorial: Development and implementation of an online, self-directed academic integrity course at Oakland University." The Journal of Academic Librarianship 38 (5): 251-258. doi: 10.1016/j.acalib.2012.06.010.

Gottardello, D., and S.F. Karabag. 2020. "Ideal and actual roles of university professors in academic integrity management: a comparative study." Studies in Higher Education: 1-19. doi: 10.1080/03075079.2020.1767051.

Gullifer, J.M., and G.A. Tyson. 2014. "Who has read the policy on plagiarism? Unpacking students' understanding of plagiarism." Studies in Higher Education 39 (7): 1202-1218. doi:10.1080/03075079.2013.777412.

Jian, H., Marion, R., \& Wang, W. (2019). Academic integrity from China to the United States: The acculturation process for chinese graduate students in the United States. Ethics \& Behavior, 29(1): 51-70. doi: 10.1080/10508422.2018.1468760.

Khanal, J., and U. Gaulee. 2019. "Challenges of international students from predeparture to post-study: A literature review." Journal of International Students 9 (2): 560-581. doi:10.32674/jis.v9i2.673.

Levine, Joy, and Vanessa Pazdernik. 2018. "Evaluation of a four-prong anti-plagiarism program and the incidence of plagiarism: a five-year retrospective study." Assessment \& Evaluation in Higher Education 43 (7): 1094-1105. doi: 10.1080/02602938.2018.1434127.

Löfström, E., T. Trotman, M. Furnari, and K. Shephard. 2015. "Who teaches academic integrity and how do they teach it?" Higher Education 69 (3): 435-448. doi: 10.1007/s10734-014-9784-3.

Macfarlane, B., J. Zhang, and A. Pun. 2014. "Academic integrity: a review of the literature." Studies in Higher Education 39 (2): 339-358.doi: 10.1080/03075079.2012.709495. 
Mahmud, S., \& Ali, I. 2021. Evolution of research on honesty and dishonesty in academic work: a bibliometric analysis of two decades. Ethics \& Behavior: 115. doi: 10.1080/10508422.2021.2015598.

Mahmud, S., and T. Bretag. 2013. "Postgraduate research students and academic integrity: 'It's about good research training'." Journal of Higher Education Policy and Management 35 (4): 432-443. doi:10.1080/1360080X.2013.812178.

Mahmud, S., T. Bretag, and T. Foltýnek. 2019. “Students' Perceptions of Plagiarism Policy in Higher Education: A Comparison of the United Kingdom, Czechia, Poland and Romania." Journal of Academic Ethics 17 (3): 271-289. doi: 10.1007/s10805-018-9319-0.

Miller, Arden, Carol Shoptaugh, and Jessica Wooldridge. 2011. "Reasons not to cheat, academic-integrity responsibility, and frequency of cheating." The journal of experimental education 79 (2): 169-184. doi: 10.1080/00220970903567830.

Newton, P. 2016. “Academic integrity: A quantitative study of confidence and understanding in students at the start of their higher education." Assessment \& Evaluation in Higher Education 41 (3): 482-497. doi:

10.1080/02602938.2015.1024199.

Obeid, Rita, and Darryl B. Hill. 2017. "An intervention designed to reduce plagiarism in a research methods classroom." Teaching of Psychology 44 (2): 155-159. doi: $10.1177 / 0098628317692620$.

Pàmies, Maria del Mar, Mireia Valverde, and Christine Cross. 2020. “Organising research on university student plagiarism: a process approach." Assessment \& Evaluation in higher education 45 (3): 401-418. doi:

10.1080/02602938.2019.1658714.

Palmer, Anne, Grace Oakley, and Mark Pegrum. 2017. "A culture of sharing: transnational higher education students' views on plagiarism in the digital era." International Journal of Management in Education 11 (4): 381-404. doi: 10.1504/IJMIE.2017.086893.

Ransome, Josie, and Philip M. Newton. 2018. "Are we educating educators about academic integrity? A study of UK higher education textbooks." Assessment \& Evaluation in Higher Education 43 (1): 126-137. doi:

10.1080/02602938.2017.1300636. 
Richards, D., S. Saddiqui, F. White, N. McGuigan, and J. Homewood. 2016. "A theory of change for student-led academic integrity." Quality in Higher education 22 (3): 242-259. doi: 10.1080/13538322.2016.1265849.

Risquez, Angelica, Michele O’Dwyer, and Ann Ledwith. 2013. “Thou shalt not plagiarise': from self-reported views to recognition and avoidance of plagiarism.” Assessment \& Evaluation in Higher Education 38 (1): 34-43. doi:10.1080/02602938.2011.596926.

Sefcik, Lesley, Michelle Striepe, and Jonathan Yorke. 2020. "Mapping the landscape of academic integrity education programs: what approaches are effective?." Assessment \& Evaluation in Higher Education 45(1): 30-43. doi: 10.1080/02602938.2019.1604942.

Smith, J.A., and P. Shinebourne. 2012. "Interpretative phenomenological analysis.” In: H. Cooper, P. M. Camic, D. L. Long, A. T. Panter, D. Rindskopf, \& K. J. Sher (Eds.), APA handbooks in psychology. APA handbook of research methods in psychology, Vol. 2. Research designs: Quantitative, qualitative, neuropsychological, and biological, 73-82. American Psychological Association.

Sotiriadou, P., D. Logan, A. Daly, and R. Guest. 2020. "The role of authentic assessment to preserve academic integrity and promote skill development and employability." Studies in Higher Education 45 (11): 2132-2148. doi: 10.1080/03075079.2019.1582015.

Sun, X., \& Hu, G. 2020. What do academics know and do about plagiarism? An interview study with Chinese university teachers of English. Ethics \& Behavior, 30(6): 459-479. doi:10.1080/10508422.2019.1633922.

Tatum, Holly, and Beth M. Schwartz. 2017. "Honor codes: Evidence based strategies for improving academic integrity." Theory Into Practice 56 (2): 129-135. doi: 10.1080/00405841.2017.1308175.

Zhang, Dongyang, Mike Joy, Georgina Cosma, Russell Boyatt, Jane Sinclair, and Jane Yau. 2014. "Source-code plagiarism in universities: a comparative study of student perspectives in China and the UK.".Assessment \& Evaluation in Higher Education 39 (6): 743-758. doi:10.1080/02602938.2013.870122. 
Zhao, X., and M. Kung. 2021. Academic Integrity of International Students:

Investigation in the Era of 'The Presumption of Innocence'. Educational Developments. 22, no. 1 . 